\title{
Signal Dependent Rician Noise Denoising Using Nonlinear Filter
}

\author{
Isshaa Aarya, Danchi Jiang, and Timothy Gale
}

\begin{abstract}
MR images are increasingly used for diagnostic and surgical procedures, as they offer better soft tissue contrast and advanced imaging capabilities. Similar to other imaging modalities, MR images are also subjected to various forms of noises and artifacts. The noise affecting MRI images is known as Rician noise and displays a nonlinear and signal dependent behavior. In this paper we propose a nonlinear filtering method for Rician noise denoising. Nonlinear filters are more capable in addressing signal dependent behavior of noise and offer good denoising with better edge preserving capabilities. A nonlinear filter based on homomorphic filter characteristics has been designed to address Rician noise in MR images. The proposed filter has been implemented on synthetic images and MR images of the articular cartilage. The efficiency of the proposed filtering method is verified by computing the PSNR and SSIM index of the image. The proposed nonlinear filter performs good denoising with improvement in the image quality as observed from the PSNR values of the image. It also offers edge preservation and can be used for both structural MRI and soft tissue study effectively
\end{abstract}

Index Terms-Homomorphic filters, MRI denoising, Rician noise, signal dependent filtering.

\section{INTRODUCTION}

The noise affecting MR images is known as Rician noise. This noise is introduced because of the magnitude image formation of MRI data and follows a Rice distribution function [1]. MRI images are converted to magnitude images and phase images by using magnitude and phase details from the k-space data obtained during image acquisition, respectively [1], [2]. The noise in k-space MRI data is usually assumed to be Gaussian white noise with zero mean [1], [2]. This reconstruction of the raw MRI data in to magnitude image; results in nonlinear behaviour of noise. It also affects both the SNR and the contrast of the MR image and makes noise dependent on the signal [2]. A wide variety of filters and filtering procedures have been studied to obtain adequate denoising in presence of Rician noise. Denoising techniques using wavelets, non-local means, median filters and anisotropic diffusion filters have all been previously suggested for Rician noise removal. The non-local means method is primarily designed for Gaussian noise removal and makes use of local neighbourhood within the image for filtering [3]. It computes Gaussian weights by using Euclidean distance between similar intensity patches within the image to perform denoising [3]. Non-local means filtering does not consider noise in the image to be Rician and

Manuscript received April 30, 2013; revised July 5, 2013.

The authors are with School of Engineering, University of Tasmania, Hobart, TAS-7001, Australia (e-mail: isaarya@ utas.edu.au, danchi.jiang@utas.edu.au,T.Gale@utas.edu.au). can cause excessive blurring if filtering parameter is not correct [3]. Anisotropic diffusion filters proposed by Perona and Malik are nonlinear filters and work by using local intensity within the homogenous regions for smoothing [4].The smoothing operation is controlled by a diffusion process, obtained from partial differential equation of heat and stops in the presence of an edge, thus preserving edge details [4]. Median filters too are nonlinear filters and make use of local statistics for noise removal, but are found to be more suitable for suppressing impulsive noise [5]. Median filters and anisotropic diffusion filters are both nonlinear filters with good edge preserving capabilities.

Most of the above mentioned procedures do not consider the effect of dependent noise on the signal while filtering. Hence even though these procedures offer sufficient denoising they are not able to isolate noise from the true MR signal and may face limitation while estimating true signal intensity, when denoising. We know that Rice noise due to their signal dependent nature modifies the true intensity of the signal and changes contrast levels in the image. Hence, in this paper we will address Rician denoising based on signal dependent behaviour of noise with help of nonlinear filters. Section II will briefly describe signal dependent filters and noise, while Section III will explain the proposed denoising method with simulation results in Section IV, followed by conclusion in Section V.

\section{SignAL-DePEndENT FILTER AND NoISE}

Our investigation of the signal dependent behaviour of Rice noise brought us to homomorphic filters for nonlinear filtering of noise [6]. Homomorphic filters are nonlinear filters in the spatial domain [6]. They require a transformation function, to separate signal and noise in the image, thus making noise linear and independent of signal [6]. Noise in this form is no longer signal dependent and can be easily filtered from the image with existing denoising procedures [6]. Homomorphic filters also require an equivalent inverse transformation function to return the filtered data back to the original equation [6]. A similar concept has been developed to make Rician noise independent of the true signal intensity. Once the noise has been made independent of the signal it undergoes filtering for noise removal and later the filtered data is transformed back into the magnitude equation. This process leads to betters estimation of the true signal intensity and is computationally more efficient.

Noise can only be considered independent of signal, if its probability distribution function is no longer dependent on the signal parameter and it can be written in a linear form [7]. Signal dependent noise can be broadly classified into 
multiplicative noise such as the speckle noise and signal-modulated additive noise such as film grain noise [6], [7]. Equation (1) represents multiplicative signal dependent noise known as speckle noise which commonly affects ultrasound images [7]. The "additive signal-modulated noise" model is a general model developed by John Walkup et.al to represent signal dependent behaviour of noise and is given by equation (2) [7]. Where, ' $r$ ' is the observed signal, ' $s$ ' is the true signal, $f(s)$ is the signal function and ' $n$ ' is noise.

$$
\begin{aligned}
& r=s \times n \\
& r=s+k \times f(s) \times n
\end{aligned}
$$

Equation (2) is based on the assumption that the noise is stationary with zero mean, uncorrelated with the signal function and represents linear signal dependent noise [7]. Noise in MRI magnitude equation is also very similar to equation (2); where noise is both signal dependent and additive. At the same time Rician noise is also nonlinear due to magnitude image formation, from information of real and imaginary components of the signal with respect to noise [8].

$$
\begin{gathered}
M=\sqrt{\left(A+n_{r}\right)^{2}+n_{i}{ }^{2}} \\
P(M \mid A, \sigma)=\frac{M}{\sigma^{2}} \exp -\frac{\left(A^{2}+M^{2}\right)}{2 \times \sigma^{2}} \times I_{o}\left(\frac{A \times M}{\sigma^{2}}\right)
\end{gathered}
$$

Equation (3) represents the magnitude image equation for MRI images and equation (4) represents the Rice distribution function for magnitude images [8]. Where, A represents true signal intensity, $n_{r}$ the real component of noise, $n_{i}$ the imaginary component of noise, $M$ the observed intensity and $I_{\mathrm{o}}$ is modified Zero-order Bessel function. If we solve the quadratic form of equation (3) we can observe that noise is still signal dependent and nonlinear. Hence, a nonlinear filter is required for denoising of magnitude MR images.

\section{Proposed DENOISING Procedure}

We propose a signal dependent filtering procedure, for Rician noise using magnitude equation given by (3). The proposed filter design tries to decouple noise from the true signal function, before filtering for noise removal [9]. It is then followed by an intuitive filtering process which tries to minimize error due to noise, using mean square estimate process using Wiener filter, on the observed noisy signal. Such a denoising operation tries to maintain the fidelity of the image without introducing any artifacts due to the filtering process and does not cause any considerable change to the original signal function. This filtered data is then used for reconstructing a noise free estimate of the image by using an inverse transformation operation. This denoising process is divided into two stages; the first stage involves transformation of nonlinear magnitude equation to make noise independent of the signal and the second stage involves filtering of the noise followed by an inverse transformation of the filtered signal; together they form a nonlinear homomorphic filter for signal dependent noise [9]. The process is explained in more detail in the following sub-sections. Fig. 1 indicates the flow chart for the proposed filter method.

\section{A. Transformation of Image}

Transformation operation is performed on the image to separate noise from the signal [9]. This transformation operation can involve any mathematical procedure such as square, logarithmic, Laplace transform, Wavelet transform etc. to simplify a nonlinear equation. Such a transformation operation enables existing image enhancement procedures to be implemented on the image [9].

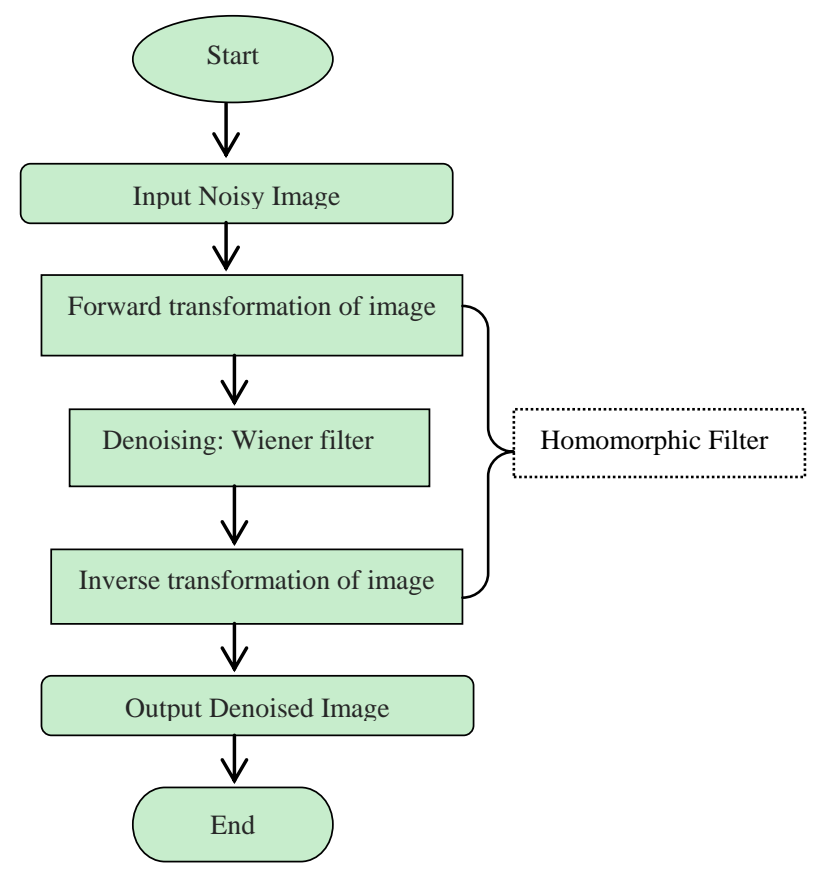

Fig. 1. Flow chart for the denoising procedure.

A similar transformation function is implemented on the Rice magnitude equation, given by equation (3). This transformation operation involves taking square of the magnitude equation, followed by a logarithmic operation. The square operation is performed on the magnitude equation so that one can gain access to individual signal and noise components and is shown by equation (6).

$$
\begin{gathered}
M^{2}=\left(A+n_{r}\right)^{2}+n_{i}^{2} \\
M^{2}=A^{2}+2 \times A \times n_{r}+n_{r}^{2}+n_{i}^{2}
\end{gathered}
$$

Estimation of noise in MR image can be determined from the background pixels using second order moment of the Rice distribution function [10].

$$
E\left[M^{2}\right]=A^{2}+2 \times \sigma_{n}^{2}
$$

Equation (7) gives us the second order moment of the Rice distribution function [10]. Due to absence of true intensity signal in the background of the MR image, the Rice distribution function follows a Rayleigh distribution and the value of true signal intensity $A$, is equal to zero in the background [10]. An estimate of noise variance in the image can then be computed by using equation (8) [10]. 


$$
\hat{\sigma}_{n}^{2}=\frac{1}{2 N} \sum_{i=1}^{N} M_{i}^{2}
$$

This variance is assumed by us, to be similar to the sum of the real and imaginary components of Gaussian noise and is subtracted from the magnitude image to reduce the overall effect of noise component in the image as shown in equation (10).

$$
\begin{gathered}
\hat{\sigma}_{n}^{2} \cong N^{2} \cong n_{r}^{2}+n_{i}^{2} \\
M^{2}-N^{2}=A^{2}+2 \times A \times n_{r}
\end{gathered}
$$

Let $M^{2}-N^{2}=\dot{M}$,

$$
\dot{M}=A \times\left(A+2 \times n_{r}\right)
$$

In equation (11), A represents the true signal intensity of the MR image, $\dot{M}$ is the observed data with overall reduced noise variance and $n_{r}$ is the real component of noise still present in the image. Equation (11) is simplified further by a logarithmic transformation. The logarithmic operation helps to separate the signal and noise component, thus reducing the magnitude equation into a linear form and decoupling noise from the signal [9]. Equation (12) is further used for the filtering process.

$$
\log (\dot{M})=\log A+\log \left(A+2 \times n_{r}\right)
$$

\section{B. Denoising Procedure}

Equation (12) can be rewritten as shown below and is similar to additive linear noise present in the image.

$$
y=x+n
$$

where $\mathrm{y}$ is the observed noisy image given by $\log (\dot{M}), x$ is the desired noise free signal and is equivalent to $\log A$ and $n$ is the noise function and represents $\log \left(A+2 \times n_{r}\right)$.

Wiener filter is now used for the denoising process, as it is more efficient in removing linear additive noise [11]. Wiener filter tries to minimize mean square error of the image function by estimating the noise free signal component from the observed signal as shown by equation (14) [11].

$$
\min \left(e^{2}\right)=[x-\hat{x}]^{2}
$$

where, $x$ is the desired noise free signal and $\hat{x}$ is the output of the Wiener filter with observed noisy data given by equation (15) as shown below,

$$
\hat{x}=W^{T} y
$$

The expectation of the mean square error output due to Wiener filter can be written as follows [12],

$$
\begin{gathered}
E\left[e^{2}\right]=E\left[\left\{x-W^{T} y\right\}^{2}\right] \\
E\left[e^{2}\right]=E\left[x x^{T}\right]-2 W^{T} E[x y]+W^{T} E\left[y y^{T}\right] W
\end{gathered}
$$

$$
E\left[e^{2}\right]=R_{x x}-2 W^{T} r_{x y}+W^{T} R_{y y} W
$$

From equation (18), $R_{x x}$ is the auto-correlation function of desired signal, $r_{x y}$ is the cross-correlation function of desired signal and the observed signal, $R_{y y}$ is the auto-correlation function of observed signal, ' $W$ ' represents Wiener filter and ' $E$ ' is the expectation operator [12]. The optimal solution for equation (18) exists when the gradient of equation (18) with respect to filter coefficient is zero [12]; thus minimizing error between desired signal and observed signal.

$$
\begin{gathered}
\frac{\partial}{\partial W} E\left[e^{2}\right]=-2 r_{x y}+2 W^{T} R_{y y}=0 \\
W^{T} R_{y y}=r_{x y} \\
W_{o p t}=\frac{r_{x y}}{R_{y y}}
\end{gathered}
$$

Equation (21) represents optimal Wiener filter for linear additive noise [12]. For additive noise given by equation (2), noise has zero mean and is uncorrelated with signal function. Hence, the cross-spectral density of desired and noisy signal can be replaced with the cross-spectral density of desired signal, such that $r_{x y}=r_{x x}$ [12]. Wiener filter in frequency domain is used for purpose of denoising and is given by equation (22) where $P_{x x}$ is the power spectral density of the desired signal with respect to power spectral density of observed signal [12], [13]. $\hat{x}(u, v)$ is an estimate of true image data obtained after convolution of Wiener filter with the degraded image in the frequency domain [12]. $H(u, v)$ in equation (22) is the point spread function which accounts for image degradation during image acquisition process and is also known as the blur operator of the filter; and $\hat{H}(u, v)$ is complex conjugate of $H(u, v)$ [12], [13].

$$
\begin{gathered}
W(u, v)=\frac{\hat{H}(u, v) \times P_{x x}(u, v)}{|H(u, v)|^{2} P_{x x}(u, v)+P_{n n}(u, v)} \\
\hat{x}(u, v)=W(u, v) \times Y(u, v)
\end{gathered}
$$

A $5 \times 5$ Gaussian blur kernel is used as point spread function for the filter, as the source noise in k-space is considered to be Gaussian in nature. Gaussian kernel can be of any size and depends entirely on the end user, although a larger kernel helps in a faster simulation process. Wiener filter too requires a prior knowledge of spectral density of true signal and noise for optimal filtering [13]. The power spectral density of Gaussian white noise is a constant flat spectrum [7], [13] and for the filtering process is estimated from background of the transformed image using equation (9). The spectral density of true signal in practice is not known, but can be estimated in different ways [13], [14]. For the given filtering process we have used the periodogram of the observed signal as an estimate of the power spectral 
density of true image [13], [14]. Equation (24) represents power spectral density of the periodogram of the observed image, where $F$ and $\hat{F}$ represent Fourier transform and conjugate of Fourier transform of the observed signal $y$ respectively and $F^{-1}$ indicates the inverse Fourier transform [14], [15].

$$
P_{x x}=F^{-1}[F(y) \times F(y)]
$$

The filtered image undergoes inverse transformation to return the image data back to the magnitude equation, with the reduced effect of noise component. For our inverse transformation we have calculated exponential of the filtered data followed by a square root operation. Equations (25) \& (26) indicate inverse transformation of the image data.

$$
\begin{aligned}
& \hat{M}=\exp (\hat{x}) \\
& \hat{A}=\sqrt{\hat{M}}
\end{aligned}
$$

The result of the denoising process is shown in Fig. 2, Fig. 3 and Fig. 5. One can also use other filters instead of Wiener filter for denoising depending on the application of the user. If the noise had been multiplicative with the signal intensity it would have been more appropriate to use geometric mean filter which is more useful in denoising signal dependent multiplicative noise [10].

\section{ILLUSTRATIVE SIMULATION}

The above filtering procedure was implemented on MR images of the articular cartilage of the knee and spine image. MR images of the articular cartilage of knee were obtained using T2-weighted fat suppressed imaging sequences. These MR images were obtained from Menzies Research Institute database, Tasmania, Australia, while the image of the spine is a commercially available image obtained from MATLAB toolbox, both of which were used for the purpose of the simulation study. A total of $62 \mathrm{MR}$ image slices of the articular cartilage were used to test the denoising procedure in presence of soft-tissue structure. Primarily histogram, PSNR, MAE and SSIM [16] were used to estimate the image quality after the denoising process. Filtered images of the spine and articular cartilage from Fig. 2 and Fig. 3 respectively, indicate that we have obtained considerable denoising for Rice noise. Also, a visual comparison of the histogram of the true image Fig. 2 b) and filtered image Fig. 2 f) of the spine, closely resemble each other indicating adequate denoising.

As predicted, nonlinear filter does not cause excessive blurring of the edges in the image or of the cartilage tissue. The denoising procedure also shows considerable improvement in the contrast levels of the filtered image. The noisy and filtered images for MRI slices 05, 21, 43 and 50 of the articular cartilage dataset are shown in Fig 5. Table I. shows a comparison results between the filtered and noisy images of the knee cartilage and spine for the proposed denoising procedure. Both the noisy and filtered images of the spine have been compared to the true image, for determining PSNR and SSIM index [16]. The filtered cartilage image has been compared with the observed image, due to absence of true noise free image of the knee. The noise estimated in the knee MRI from the background is of standard deviation 13.54 and that for the spine image is 6.69 using equation (8).

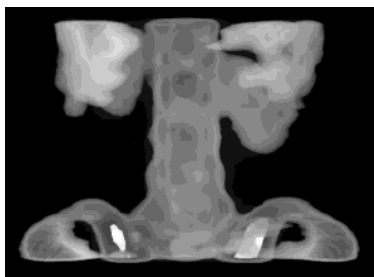

a)

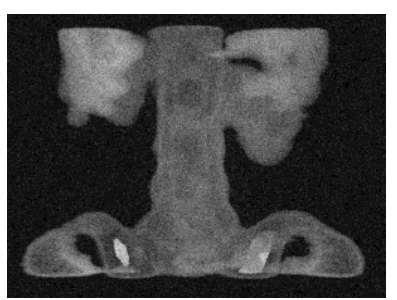

c)

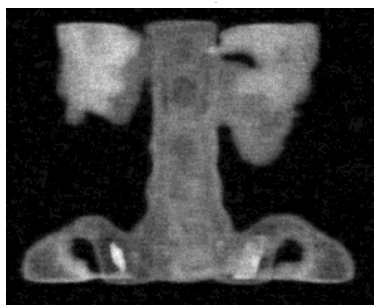

e)

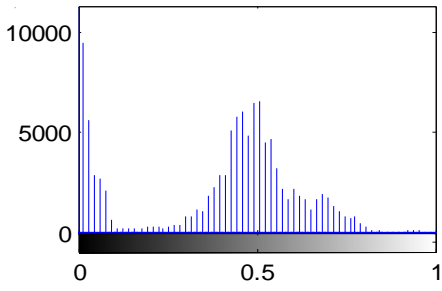

b)

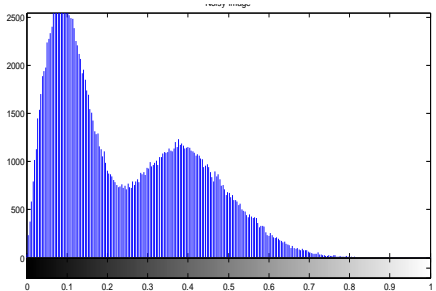

d)

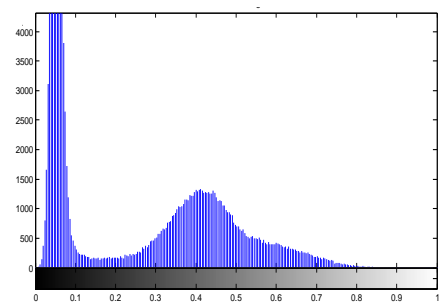

f)
Fig. 2. a) True noise free image of the spine b) histogram plot of true noise free image c) image with standard deviation of noise $=7$ d) histogram plot of noisy image e) Denoised image using nonlinear filtering and f) histogram plot of filtered image which is very similar to orginal histogram.

TABLE I: COMPARISON OF IMAGE PARAMETERS

\begin{tabular}{llll}
\hline \hline Images & $\begin{array}{l}\text { Knee } \\
\boldsymbol{\sigma}=13.54\end{array}$ & $\begin{array}{l}\text { Spine Noisy } \\
\boldsymbol{\sigma}=6.69\end{array}$ & Spine filtered \\
\hline PSNR & 27.1173 & 29.6466 & 34.7226 \\
SNR & 21.8546 & 8.8277 & 23.4107 \\
MSE & 126.2842 & 70.538 & 21.9191 \\
MAE & 8.7230 & 6.9826 & 4.0471 \\
SSIM & 0.5910 & 0.3844 & 0.6201 \\
\hline \hline
\end{tabular}

A plot of PSNR versus standard deviation of noise was also prepared for study of the denoising process at higher values of noise, using the spine image. The original spine image was subjected to noise of different standard deviation and a plot of PSNR of the filtered and noisy image was computed for the corresponding standard deviation of noise.

The PSNR value of the filtered image was found to be higher than that of the noisy image and decreased as the standard deviation of noise increased along the horizontal axis as shown in Fig. 4.

The noise estimation in the proposed filter design has limitations when zero-intensity background is absent in the image. Also, we observe that the proposed transformation function does not make noise completely independent of the signal, which could still lead to insufficient denoising. Future work may include a modified Wiener filter design with a 
more accurate estimation of true noise and a better nonlinear transformation function to make noise truly independent of the signal for MRI magnitude equation.

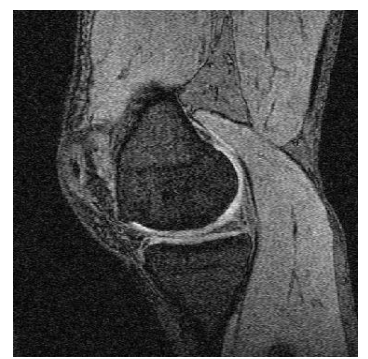

a)

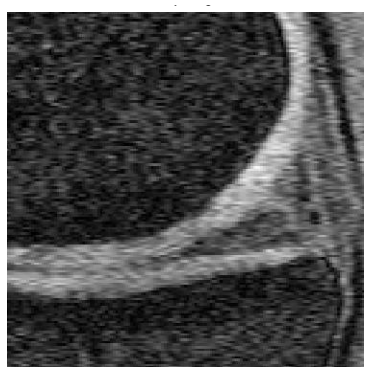

c)

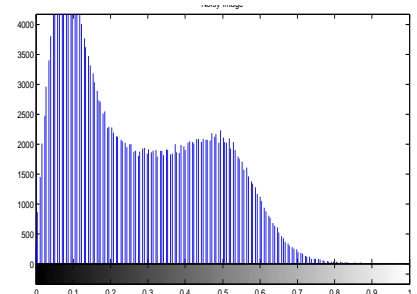

e)

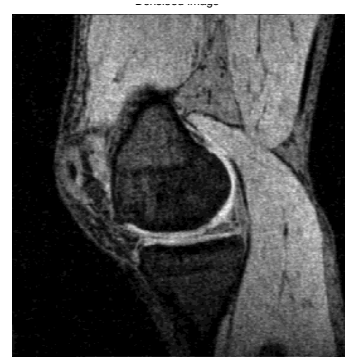

b)

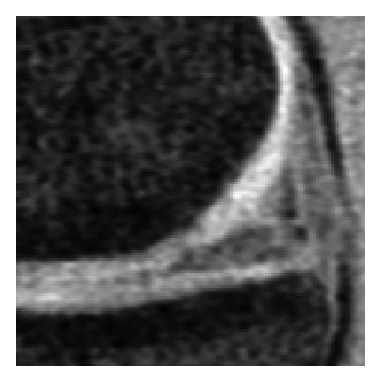

d)

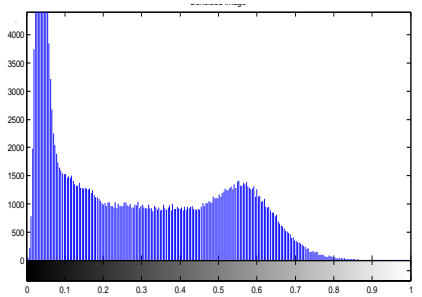

f)

Fig. 3. a) Noisy MRI image of articular cartilage, slice 16 of MRI dataset. b) Denoised cartilage image c) magnified image of cartilage tissue d) magnified image of denoised cartilage tissue e) histogram of noisy image and f) histogram of filtered image.

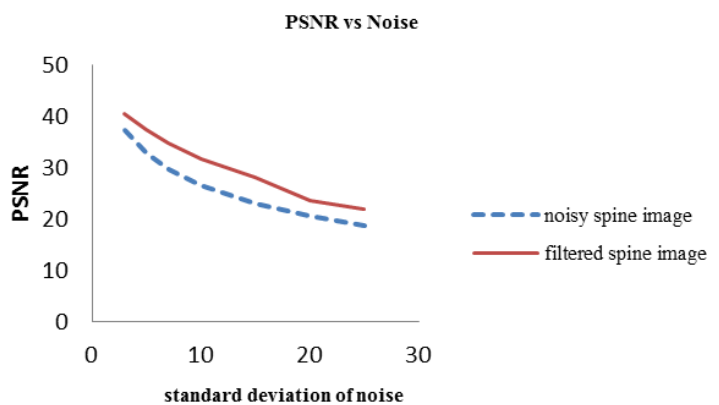

Fig. 4. Plot of PSNR versus standard deviation of noise for spine image.

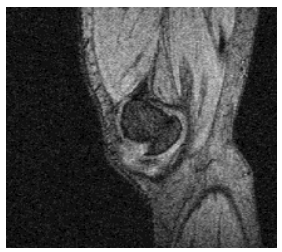

a)

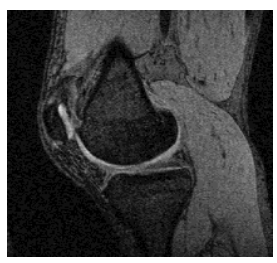

c)

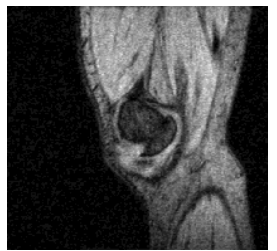

b)

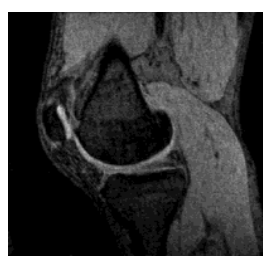

d)

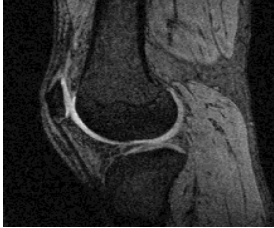

e)

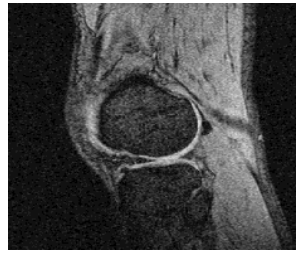

g)

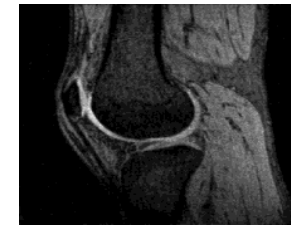

f)

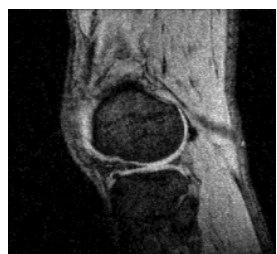

h)
Fig. 5. a) observed image slice 05 and b) denoised images slice 05 , c) observed image slice 21 and d) denoised images slice 21 , e) observed image slice 43 and $\mathrm{f}$ ) denoised images of slice 43 and $\mathrm{g}$ ) observed image slice 50 and $\mathrm{h}$ ) denoised images of slice 50 of articular cartilage of the knee.

\section{CONCLUSION}

In this paper, a nonlinear filter based denoising procedure has been proposed from our observation of the advantages offered by homomorphic filter and Weiner filter. An intuitive reasoning process offered by Wiener filter has been included to deduct the denoising procedure. The simulation results show the efficiency of the proposed method for signal dependent filtering of Rician noise. It can also be observed that the proposed filter helps in better preservation of edge details. As such, it can be used in soft tissue denoising where extreme loss of edge details can be prevented while performing denoising or other image enhancement procedures.

The future research focus can include a more advanced implementation of the filter design using a suitable image transformation to make noise completely independent of the signal and an even more accurate estimation of noise for a more efficient denoising procedure with improved contrast and edge preservation capabilities.

\section{REFERENCES}

[1] R. D. Nowak, "Wavelet-based rician noise removal for magnetic resonance imaging," IEEE Trans. Image Process, vol. 8, no. 10, pp. 1408-1419, Oct. 1999.

[2] H. Gudbjartsson and S. Patz, "The rician distribution of noisy MRI data," Mag Reson Med., vol. 34, no. 6, pp. 910-914, December 1995.

[3] A. Buades, C. Bartomeu, and J. Morel, "A non-local algorithm for image denoising," in Proc. IEEE Computer Society Conf. CVPR 2005 , vol. 2, June 2005, pp. 60-65.

[4] P. Perona and J. Malik, "Scale-space and edge detection using anisotropic diffusion," IEEE Trans. Pattern. Anal. Machine. Intell., vol. 12, no. 7, pp. 629- 639, Jul. 1990.

[5] L. Yin, R. Yang, M. Gabbouj, and Y. Neuvo, "Weighted median filters: A tutorial," IEEE Trans Circuits Syst. II, Analog Digit. Signal Process 2, vol. 34, no. 3, pp. 157-192, Mar. 1996.

[6] H. Arsenault and M. Denis, "Image processing in Signal-Dependent noise," Can. J. Phys., vol. 61, no. 2, pp. 309-317, 1983.

[7] J. F. Walkup and R. C. Choens, "Image processing in signal-dependent noise," Optical Engineering, vol. 13, no. 3, pp. 258-266, Jun. 1974.

[8] J. Sijbers, A. den Dekker, P. Scheunders, and D. Dyck, "Maximum-likelihood estimation of rice distribution parameters," IEEE Trans. Med. Imag., vol. 17, no. 3, pp. 357-361, June 1998.

[9] I. Pitas and A. N. Venetsanopoulos, "Nonlinear mean filters in image processing," IEEE Trans. Acoust., Speech, Signal Process, vol. 34, no. 3, pp. 573- 584, Jun. 1986.

[10] S. Aja-Fernandez, C. Alberola-Lopez, and C. Westin, "Noise and signal estimation in magnitude MRI and rician distributed images: A 
LMMSE approach," IEEE Trans. Image Process, vol. 17, no. 8, pp. 1383-1398, Aug. 2008.

[11] R. Gonzalez, R. Woods, and S. Eddins, Digital Image Processing, Upper Saddle River, N.J: Pearson Prentice Hall, 2004, ch. 5, pp. $170-173$

[12] S. Vaseghi, Advanced Digital Signal Processing and Noise Reduction, 2nd ed., Copyright (C) 2000, John Wiley and Sons, ch. 6, pp. 179-183/189-196, ISBN, 0-470-84162-1.

[13] J. S. Lim, Two-Dimensional signal and image Processing, Copyright $\odot$ 1990, New Jersey, US: Prentice Hall PTR, ch. 6 pp. 349-352, 354-356, 363 and ch. 9, pp. 527, 544-568.

[14] M. Banham and A. Katsaggelos, "Digital image restoration," IEEE Signal Processing Mag., vol. 14, no. 2, pp. 24-41, Mar. 1997.

[15] M. Mehri, P. Gomez-Kramer, P. Heroux, and R. Mullot, "Old document image segmentation using the autocorrelation function and multiresolution analysis," Document Recognition and Retrieval, vol. XX, Feb. 2013.

[16] Z. Wang, A. Bovik, H. Sheikh, and E. Simoncelli, "Image quality assessment: From error visibility to structural similarity," IEEE Trans. Image Process, vol. 13, no. 4, pp. 600-612, Apr. 2004.

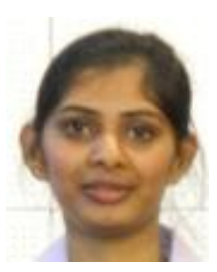

Isshaa Aarya has received her MSc. in biomedical engineering from University of Dundee, UK in 2011 and B.E in Biomedical Engineering from University of Mumbai, India in 2009. She is currently a PhD student with University of Tasmania, Australia. Her research interests include medical image processing and 3D modelling for patient specific diagnosis and non-invasive surgeries. She is an IEEE student member.

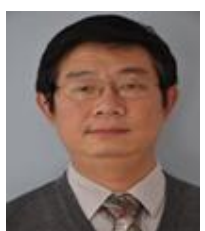

Danchi Jiang is a senior lecturer in telecommunication engineering and chair of computer systems engineering stream at the School of Engineering, University of Tasmania. He has obtained his $\mathrm{PhD}$ in Systems Engineering from Australian National University and conducted research in the area of intelligent system and telecommunication at Chinese University and Hong Kong and National ICT Australia (NICTA). His main research interests are in the area of advanced multidimensional signal processing with applications in telecommunication, biomedical engineering and micro-grid power systems.

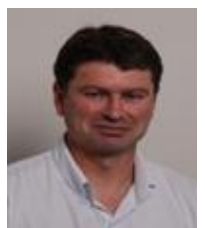

Timothy Gale is currently a senior lecturer in biomedical and mechatronics engineering at the University of Tasmania. His research interests include modelling, simulation, signal processing and control in medical areas that include neonatal physiology, motorised prosthetics and medical imaging. He also serves as a Biomedical College Board Member and Chair of the National Committee on Mechatronics for Engineers Australia. 